\title{
Sistemas de protección social emergentes en el contexto de las reconfiguraciones del mundo del trabajo y de las nuevas manifestaciones de la cuestión social
}

\section{Emerging social protection systems in the context of the reconfigurations of the world of work and the new manifestations of the social issue}

\author{
Susana Beatriz Moniec ${ }^{a}$
}

\begin{abstract}
Resumen
El pasaje del régimen Fordista al Postfordista implicó modificaciones en la protección social pensada en términos de Estados de Bienestar Social. En contraposición emergen nuevos enfoques como el Estado Social activo (ESA), el Work Fare, el de Asistencia y Promoción y el Manejo Social del Riesgo, entre otros, que pretenden dar cuenta de los procesos de re-mercantilización de la fuerza de trabajo que el nuevo régimen de acumulación impone.

En este artículo se presenta en primer término un análisis comparado de los supuestos y principios de estos enfoques de protección social. $\mathrm{Al}$ respecto, se hipotetiza que los mismos implican modificaciones en el rol del Estado y de los propios sujetos, hacia quienes se trasfiere la responsabilidad por su propia protección social, abonándose procesos de re individualización, a partir de la promoción del auto reciclaje, del emprendedurismo, del asociativismo. Finalmente en el artículo se concluye que estos sistemas de protección emergentes omiten el tratamiento de las relaciones de producción capital trabajo, suponiendo que el problema de la pobreza está vinculado fundamentalmente a las falta de activos de los sujetos implicados en situaciones de pobreza y desigualdad.
\end{abstract}

Palabras clave: enfoques de protección, políticas sociales, subjetividades.

\footnotetext{
Abstract

The passage from the Fordist regime to the Postfordist one implied modifications in the social protection thought in terms of Social Welfare States. In contrast, new approaches emerge such as the Active Social State (ESA), the Work Fare, the Assistance and Promotion and Social Risk Management, among others, which aim to account for the processes of re-commodification of the workforce
}

Kera Yvoty: reflexiones sobre la cuestión social. Vol. 2, 2017, 9-15.

ISSN (impreso): 2519-7797

a Universidad Nacional de Misiones, Facultad de Humanidades y Ciencias Sociales, Departamento de Trabajo Social, Argentina.

Correspondencia a: sumoniec@gmail.com

Cita:

Moniec, S. (2017). Sistemas de protección social emergentes en el contexto de las reconfiguraciones del mundo del trabajo y de las nuevas manifestaciones de la cuestión social. Kera Yvoty: reflexiones sobre la cuestión social, 2, 9-15.

Recibido:

2 noviembre 2017

Aceptado:

20 diciembre 2017 
that the new regime of accumulation imposes.

In this article, a comparative analysis of the assumptions and principles of these social protection approaches is presented first. In this regard, it is hypothesized that they imply changes in the role of the State and of the subjects themselves, to whom responsibility for their own social protection is transferred, with re-individuation processes being paid, based on the promotion of self-recycling, entrepreneurship, of associativism. Finally, the article concludes that these emerging protection systems omit the treatment of capital - labor production relations, assuming that the problem of poverty is fundamentally linked to the lack of assets of the subjects involved in situations of poverty and inequality.

Keywords: protection approaches, social policies, subjectivities.

\section{Introducción}

Para Harvey (1998) la fragmentación de la configuración fordista-keynesiana de posguerra, dio origen a un periodo de cambios e incertidumbres, que el autor lo asocia a la emergencia de un nuevo régimen de acumulación y su correspondiente modo de regulación social y política.

La reconfiguración de relaciones de fuerza que la transición hacia la acumulación flexible (Harvey, 1998) o de alto valor (Reich, 1993) produjo, invalidó las condiciones que originaron el equilibrio de poder que sostenía el contrato social global tácito (Harvey 1998), entre el capital, el Estado y el trabajo organizado.

Esta nueva forma de organización de la producción, tendiente a abordar las contradicciones del capitalismo, que se caracteriza por apelar "a la flexibilidad con relación a los procesos laborales, los mercados de mano de obra, los productos y las pautas de consumo (...); que entraña la compresión espacio-temporal" (Harvey, 1998, p. 170-171), nos transformó como sociedad, colocando en jaque a las instituciones de la modernidad.

Corrientemente observamos en relación al mundo del trabajo, en particular en el caso de Argentina, la extensión de la situaciones de temporalidad, de subcontratación en el empleo, así como el ensanchamiento de la categoría monotributistas, la de los trabajadores autónomos o de aquellos subsidiados por el Estado mediante transferencias monetarias; todas situaciones que hace dos décadas atrás eran consideradas atípicas, pero que en la actualidad progresivamente se imponen, desplazando a la categoría del asalariado que gozaba de estabilidad en el empleo y estaba protegido por el régimen de seguridad social y derechos sociales, vinculado a su status de trabajador.

Estas nuevas disposiciones en el mundo del trabajo y en la estructura del mercado laboral, definidos por la prominencia de regímenes de empleo flexible, impactan en los sistemas de protección social' ${ }^{1}$ e implican una clara remercantilización de la fuerza de trabajo, en base a lo cual distintos autores han planteado la emergencia de nuevas manifestaciones de la cuestión social que tensionan la cohesión social.

Así, la nueva cartografía social impuesta por el desarrollo del capitalismo en el Siglo XXI, tipificada por la extensión de la vulnerabilidad de masas, interpela a los sistemas de protección social gestados en el marco del régimen fordista de acumulación y propicia, en el ámbito de

\footnotetext{
1 Sistemas que a su vez se encuentran interpelados en su sostenibilidad económico financiera en el tiempo, en razón del aumento de la expectativa de vida; por la relación decreciente entre activos-pasivos; por las moderadas tasas de crecimiento; por los nuevos riesgos que emergen en la sociedad pos fordista y por los nuevos movimientos y sectores sociales que cuestionan su dominio sobre la esfera de la sociedad civil y la necesidad de contemplación de otros tipos de derecho.
} 
las ciencias sociales y en particular de los gobiernos y organismos internacionales, la discusión sobre las actuales expresiones de cuestión social, marcando la necesidad de pensar nuevas alternativas de intervención para su abordaje.

\section{Esquemas de protección social emergentes}

Entre los nuevos esquemas que se comienzan a diseñar para el abordaje de la problemática de la inserción y la reproducción de la vida de las poblaciones, emergen en escena modelos, enfoques y marcos normativos para la protección social tales como: Workfare (Boyer, 2006), Estado Social Activo (Boyer, 2006), Manejo Social de Riesgo (Holzmann \& Jorgensen, 2003) y el de Asistencia y Promoción (Cecchini \& Martínez, 2011), que proponen formas de intervención en lo social que se diferencian de aquellas propiciadas desde los estados sociales característicos del régimen fordista-keynesiano, interpelando con su impronta, la viabilidad económica y política del Enfoque de Derechos referenciado por Sepulveda (2014); e impactando en la relación estado - sociedad y en las posibilidades del ejercicio de la ciudadanía y de la democracia (Figura 1).
A pesar que estos modelos se perfilan para países o regiones, con distintas tradiciones políticas: liberal, conservadora o socialdemócrata (el ESA para los países de Europa, el Work Fare para Estados Unidos y Reino Unido, la asistencia y promoción para Latinoamérica), el análisis de sus narrativas da cuenta de la configuración de dispositivos de intervención que denotan elementos comunes tales como la re orientación del gasto social, la noción de riesgo que involucran, los destinatarios de las acciones, los actores que deben estar involucrados y los roles que deben asumir y, los propósitos o efectos deseados. Rasgos estos, que parecieran nutrirse de una base común ideada desde el marco conceptual para el manejo social del riesgo y que permiten anticipar la emergencia de nuevos sistemas de protección social basados en trayectorias individuales y configurados a partir de formas de protección auto gestionadas por los propios actores, que contemplen la diversidad y fragmentación de las situaciones sociales y laborales, esto a diferencia de las protecciones construidas fundadas en colectivos abstractos, característicos del fordismo (Tabla 1).

A partir del análisis de los autores trabajados en este artículo, podemos

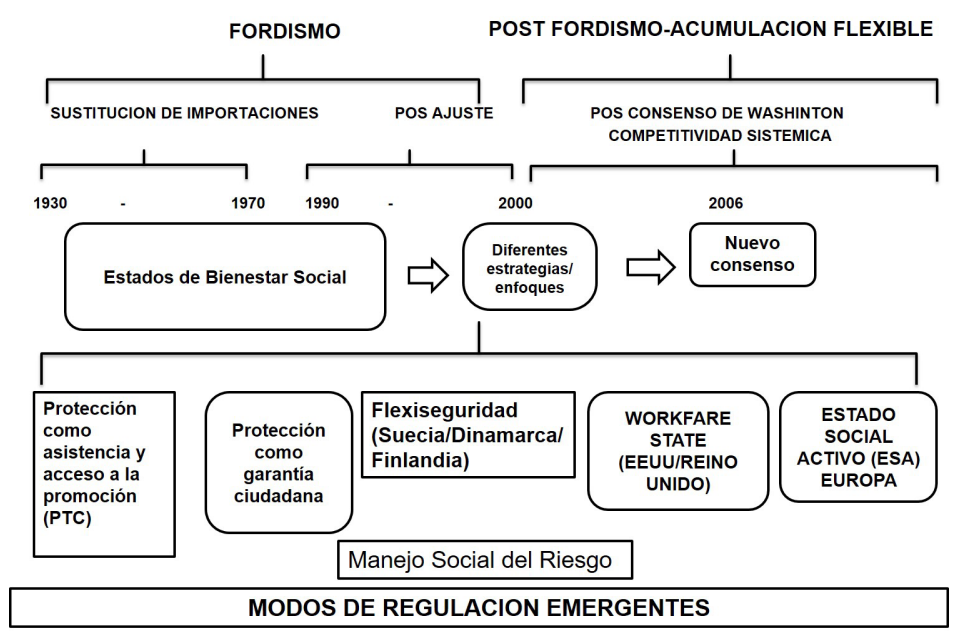

Figura 1. Enfoques de la protección social en andamiento.

Fuente: Construido en base al análisis de Boyer (2006),

Holzmann y Jorgensen (2003), Cecchini y Martínez (2011) y

Sepulveda (2014).

Kera Yvoty: reflexiones sobre la cuestión social. Vol. 2, 2017. 
Tabla 1

Roles, actores y propósitos de los enfoques de protección social emergentes

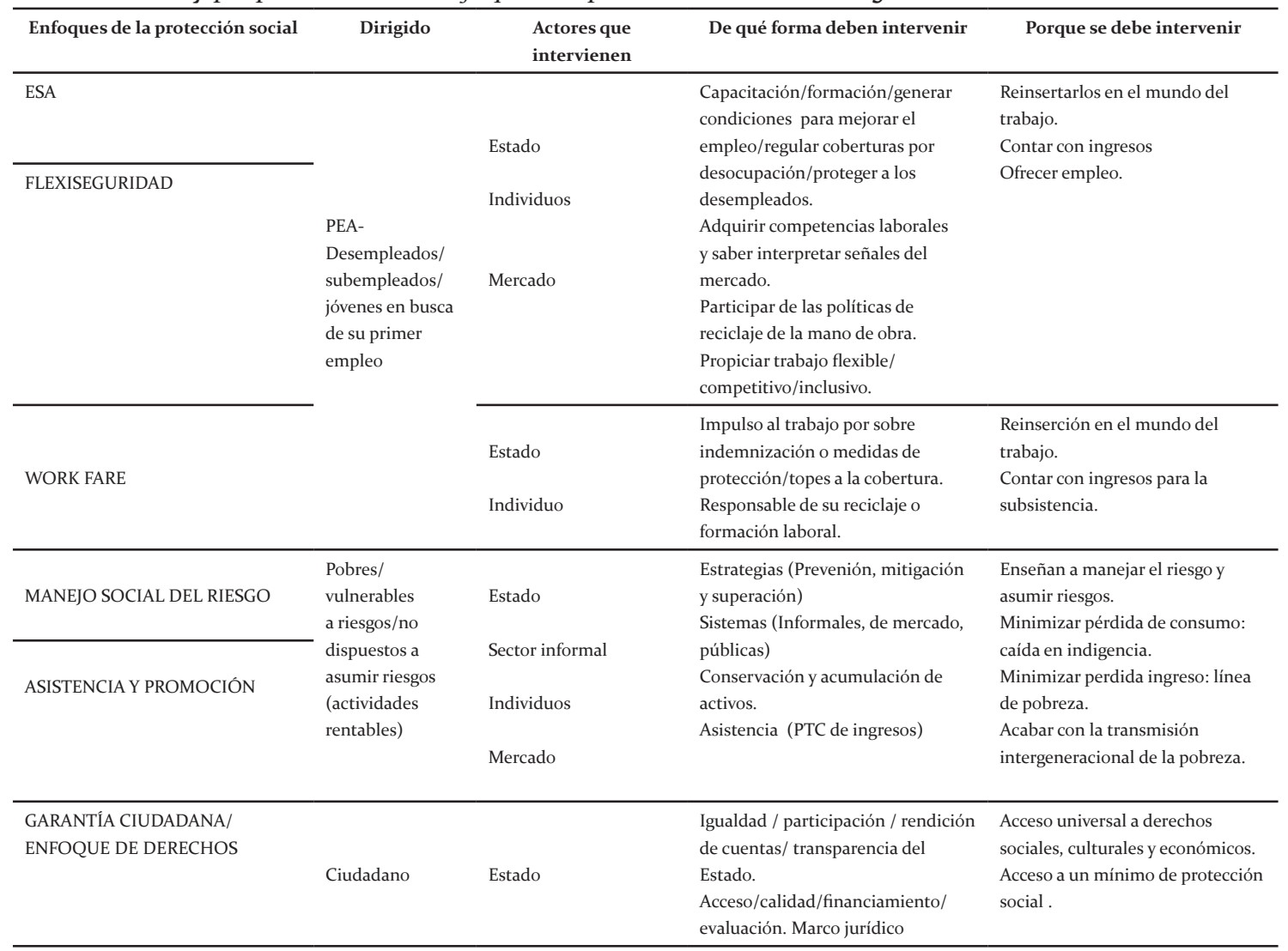

Fuente. Elaboración propia en base al análisis de Boyer (2006), Holzmann y Jorgensen (2003), Cecchini y Martínez (2011) y Sepulveda (2014).

observar que la población objeto de intervención difiere entre los enfoques. Los dos primeros, con incidencia en los países desarrollados, están dirigidos a los individuos con dificultades para insertarse en el mercado de trabajo; en tanto en el marco del Manejo Social del Riesgo y en el de Asistencia y Promoción, la población objeto son los pobres; aquellos sin capacidad empresaria o micro emprendedora como para asumir riesgos vinculados a actividades rentables.

No obstante esta distinción en las poblaciones objetos, como elemento común entre los distintos enfoques expuestos (Estado Social Activo, Work Fare, Manejo Social del Riesgo y Asistencia y Promoción), se destaca la proactividad que deben asumir los sistemas en relación al problema del desempleo extendido y la falta de ingresos de la población para atender la reproducción de sus condiciones de vida.

En torno a ello, el precepto fundamental del ESA y del Work Fare es incentivar el empleo desde diferentes frentes: replanteando el rol del Estado en relación al mercado y en su función en los sistemas de protección y modificando la finalidad de los sistemas de protección y compeliendo a los sujetos a que se reciclen y desarrollen competencias que les permitan incorporarse rápidamente al mercado de trabajo. En tanto en los otros dos, la idea fundamental se asienta en la necesidad que los sujetos desarrollen e incorporen activos 
de diverso tipo, que los tornen capaces de prevenir o minimizar los riesgos, o sortearlos una vez estos hayan ocurrido. De este modo, el manejo social del riesgo como marco conceptual interpela los sistemas informales, de mercado y públicos.

A pesar de la convergencia de los sistemas en torno al rol del estado, responsabilizándolo en el cumplimiento de funciones de asistencia y promoción en torno a los propósitos de la empleabilidad y la incorporación de activos, las formas de intervención van a diferir en función de sus configuraciones históricopolíticas liberales o socialdemócratas. Al respecto Esping Andersen (1991) plantea distinciones entre regímenes, recuperando el papel de las fuerzas históricas interactivas que están por detrás de los sistemas de protección; de este modo el modelo de formación política de la clase trabajadora, la particularidad de las coaliciones políticas y la institucionalización de las preferencias de clase y del comportamiento político, van a dar cuenta de la singularidad que asumen estos regímenes en cada país.

Así, a diferencia del ESA, en que se continua postulando que "la solidaridad debe expresarse a través de procedimientos colectivos, alternativos a la lógica mercantil" (Boyer, 2006, p. 16); el Work Fare, con su intervención buscaría alivianar el gasto social en coberturas vinculadas a la situación de desempleo, la reducción de los presupuestos sociales y consecuentemente de las coberturas y la transferencia al propio sujeto de la responsabilidad por su formación en relación a las competencias y habilidades que demanda el mercado

La reestructuración de los sistemas de protección, que promueven la integración social a través del empleo o de la incorporación de activos, va a tener implicancias en los modos de intervención social, por la redefinición de las prácticas que exige, así como por la emergencia de nuevas profesiones vinculadas a las nuevas funciones necesarias para poner en andamiento esta nueva concepción de riesgo, derivada de las dificultades de los sujetos para hacer lecturas pertinentes respecto de las necesidades del mercado y por otro para poner en andamiento la noción de activación e integración por el empleo, o mediante la asunción de actividades rentables.

La puesta en acto de modos de intervención implica el despliegue de distintas estrategias que trascienden la mediación entre recursos y necesidades. En la intervención se pone en juego la interpretación, la productividad simbólicay los procesos de producción de subjetividad que ello conlleva, y que dicen respecto de la reproducción de las relaciones sociales de producción. En ese sentido observamos que el lenguaje juega un papel importante en la construcción de significados respecto de cuáles son los problemas y cuáles las alternativas y modos de afrontarlos, pues entendemos, en términos de Austin, que se pueden "hacer cosas con palabras". (1955). Por ello, la intervención sobre lo social reviste una "eficacia simbólica, (...) contenida en la discursividad, en la gramática, en la retórica de lo social que sobre determina la intervención" (Matus, 2006, p. 37).

Incentivación, activación, micro emprendedor, emprendedurismo, micro empresario, lectura perversa o errada de los individuos respecto de las señales del mercado sobre empleos disponibles y requerimientos de competencias y habilidades: configuran enunciados que denotan la transferencia de responsabilidades hacia los sujetos por su situación de desigualdad, velando el modo en que operan las relaciones de producción capital -trabajo en este proceso, que claramente marchan en el camino del disciplinamiento y remercantilización de la fuerza de trabajo. En este sentido interesante resulta recuperar el análisis de Harvey (1998) en relación al modo en que el capitalismo del siglo XXI regula su crisis de hiperacumulación:

La acumulación flexible parece 
constituir una simple combinación de las dos estrategias básicas definidas por Marx para obtenerganancia (plusvalía). La primera, llamada plusvalía absoluta, se sustenta en la extensión de la jornada de trabajo con relación al salario necesario para garantizar la reproducción de la clase obrera en un determinado nivel de vida. El desplazamiento hacia la prolongación de las horas de trabajo, junto con una reducción global de los estándares de vida, sea por erosión de los salarios reales sea por el desplazamiento del capital de las corporaciones a regiones de altos salarios a regiones de bajos salarios. Mediantela segunda estrategia, denominada plusvalía relativa, se realiza la transformación organizativa y tecnológica con el propósito de generar ganancias temporarias para las firmas innovadoras y beneficios generalizados en la medida en que se reducen los costos de los bienes que definen el nivel de vida de la fuerza de trabajo. (Harvey, 1998, p. 210)

La remercantilización de la fuerza de trabajo, junto con la reorientación de los gastos sociales y la apelación de los sistemas de protección a la pluralidad de sectores, fundamentalmente de las familias, interpela las posibilidades de la puesta en práctica de los distintos principios que el enfoque de derechos implica (participación, accesibilidad, igualdad, transparencia, rendición de cuentas, entre otros). Enfoque fundando en convenciones internacionales, muchas de ellas suscriptas aún bajo la preeminencia del régimen Fordista, en el que el contrato social daba cuenta de un equilibro de poder entre el Estado, el capital y el trabajo, hoy claramente inclinado hacia el capital.

\section{A modo de reflexión preliminar}

Concomitante

con las transformaciones en el régimen de acumulación y en el modo de regulación social, con la emergencia de nuevos sistemas de protección, en el ámbito de las políticas sociales instrumentadas en las últimas décadas $y$ en actual funcionamiento, en particular en Argentina $^{2}$, es posible reconocer una reconfiguración en la intervención social; claramente visualizada en la expansión de políticas sociales que asumen dispositivos de intervención social basados en los supuestos de los nuevos enfoques, que responsabilizan de los sujetos por su situación de desventura y consecuentemente los compelen a involucrarse y comprometerse en el desarrollo de comportamientos, conductas y competencias necesarias para la gestión de la vida cotidiana.

El sustrato ideológico de los nuevos dispositivos de intervención, promueve la instalación y masificación de nuevas subjetividades vinculadas a la remercantilización de la fuerza de trabajo, que en el actual contexto de retracción de las protecciones construidas, anticipan la extensión de la reprimarización de la protección social, que colisiona con los principios del enfoque de derechos postulados por Sepulveda (2014); asumido por la mayoría de nuestros países a partir de la adhesión a las convenciones internacionales y de la concomitante sanción de leyes.

\section{Referencias Bibliográficas}

Boyer, R. (2006). El Estado Social a la luz de las investigaciones regulacionistas recientes. Revista de Trabajo, 2(3), 139156.

Cecchini, Simone \& Martinez, R. (2011). Protección social inclusiva en América Latina. Una mirada integral, un enfoque de derechos. Santiago de Chile: CEPAL, GIZ.

Esping Andersen, G. (1991). As tres

\footnotetext{
2 Algunos ejemplos que dan cuento de ello son los programas: PROGRESAR, Jóvenes con más y mejor trabajo, Empleo Independiente, Alimentario, entre otros).
} 
economias politicas do Welfare State. Revista Lua Nova, (24).

Harvey, D. (1998). Condição Pós Moderna. Ed. Loyola.

Harvey, D. (2004). Los nuevos rostros del imperialismo. Santiago de Chile: Centro de Estudios Miguel Enríquez. Recuperado de: http:/www.archivochile.com
Holzmann, R. \& Jørgensen, S. (2000). Manejo Social del Riesgo: Un nuevo marco conceptual para la Protección Social y más allá. Banco Mundial.

Sepulveda, M. (2014). De la retórica a la práctica: el enfoque de derechos en la protección social en América Latina. Santiago de Chile: CEPAL. 